\title{
Space-based Lasers for Remote Sensing Applications
}

\author{
Anthony W. Yu \\ NASA Goddard Space Flight Center, Laser \& Electo-Optics Branch, Greenbelt, MD 20771 \\ .anthony.w.yu@nasa.gov
}

\begin{abstract}
There are currently three operational lidar systems orbiting the Earth, the Moon and the planet Mercury gathering scientific data and images to form a better understanding of our Earth and solar system. In this paper we will present an overview of the spaceborne laser programs and offer insights into future spaceborne lasers for remote sensing applications.
\end{abstract}

OCIS codes: (280.0280) Remote Sensing and Sensors; (280.3420) Laser Sensors; (280-3640) Lidar; (140.3580) Solid State Lasers

\section{Introduction}

In the past few decades, high power laser diode arrays (LDA) have became the enabling technology that led to a series of successful spaceborne lidar system for Earth observing and planetary exploration. One of the first diode pumped solid state lasers was the Mars Orbiter Laser Altimeter (MOLA) laser transmitter launched in 1996, which produced high resolution topographic map of the planet Mars [1]. This was followed by the Geoscience Laser Altimeter System (GLAS) in 2003; the CALIOP and the Mercury Laser Altimeter in 2006; and the Lunar Orbiter Laser Altimeter in 2008 [2, 3, 4, 5]. These US-led missions as well as missions from other countries have produced vast amount of valuable scientific data for the study of our solar system. Although successful, the current DPSSL technology used for these spaceborne missions still cannot address the demanding requirements of future lidar missions.

\section{Overview of Current Spaceborne Laser Architectures}

Quasi-continuous wave (QCW) LDA pumped Nd:YAG lasers have been used since the late 1990's in all of the spaceborne lidar missions for NASA. The MOLA laser that was launched in 1996 was a diode-pumped, Nd:YAG zig-zag slab, cross-Porro, actively q-switched laser transmitter operated at $1.064 \mu \mathrm{m}$ wavelength, pulse energy of 40 $\mathrm{mJ}, 10 \mathrm{~ns}$ pulse width and repetition rate of $10 \mathrm{~Hz}$. The prime science objective of MOLA was to determine the global topography of Mars at a level suitable for addressing problems in geology and geophysics. Since the launch of MGS on November 1996 until the instrument was commanded off on June 2001, a total of 670 million shots were fired with 640 million measurements of the Mars surface and atmosphere. This represents more than ten times the number of laser measurements than all previous space lidar missions combined. The MOLA instrument performance and lifetime have surpassed all goals of the MOLA investigation [6]. The Shuttle Laser Altimeter (SLA) I \& II also flew a copy of the MOLA laser in 1996 and 1997 to produced topographic maps of the Earth surface and accumulated a total of about 6 million shots in space [7].

The GLAS laser transmitter is the first passively q-switched, master oscillator power amplifier (MOPA) design that operated in space. The instrument was launched in 2003 and completed its mission in early 2011 with a total of just under 2 billion shots in space from three transmitters [8]. The GLAS lasers represent the next generation of spacebased remote sensing laser transmitters. The GLAS lasers generally have an order-of-magnitude higher performance than MOLA in power ( $110 \mathrm{~mJ}$ total with $75 \mathrm{~mJ}$ at $1.06 \mu \mathrm{m}$ and $35 \mathrm{~mJ}$ in $0.532 \mu \mathrm{m})$, beam quality (1I0 $\mu \mathrm{rad}$ ), pulse width of $<6 \mathrm{~ns}$ and at $40 \mathrm{~Hz}$. The GLAS MOPA laser modular design provided the baseline architecture for the next two missions to the planet Mercury and the Moon.

The Mercury Surface, Space Environment, Geochemistry, and Ranging (MESSENGER) mission to the planet Mercury requires a laser altimeter capable of performing range measurements to the surface of the planet over highly variable distances and with a constantly changing thermal environment. The satellite, having just reached final orbit and began gathering data in March 2011, will execute an orbit with a periapsis of $200 \mathrm{~km}$ and an apoapsis of approximately $15,200 \mathrm{~km}$, with an orbital period of $12 \mathrm{~h}$. For the altimeter instrument, science observations are taken during the $0.5 \mathrm{~h}$ of closest approach to the planet. The laser must support the instrument requirement of achieving a range resolution of less than $40 \mathrm{~cm}$ to the surface of the planet. The anticipated radiation exposure over the mission life is $30 \mathrm{krad}(\mathrm{Si})$, total dose, assuming an effective shielding of $0.1 \mathrm{~cm}$ of aluminum. During the close approach to the day side of the planet the satellite is heating, and it is not possible to fully isolate the laser subsystem from the rest of the satellite. Mission requirements on the laser included more than $18 \mathrm{~mJ}$ of output energy in a near-diffraction-limited beam with $6 \mathrm{~ns}$ pulses at an $8 \mathrm{~Hz}$ repetition rate, while the laser bench temperature is 
executing a thermal ramp from 15 to $25^{\circ} \mathrm{C}$ at a rate of approximately $0.4^{\circ} \mathrm{C}$ min. The MLA laser transmitter was based on the GLAS MOPA laser design but with only a single stage amplifier. The oscillator was changed from a Porro/Mirror resonator to a cross-Porro resonator to ensure that the laser will maintain its alignment over the harsh environment of Mercury [9].

The first spaceborne lidar, came just ten years after the demonstration of the first laser in 1960, was flown on Apollo 15,16 and 17 and provided the first topographic mapping of the moon from the Moon's orbit. It was a flash lamp pumped, mechanically q-switched ruby laser built by RCA [10]. The Lunar Reconnaissance Orbiter (LRO) marked our return to the moon for over four decades since the Apollo era with the objectives to conduct investigations and prepare for future human exploration of the Moon, Mars and beyond. The overall mission objective for the LRO is to find and locate landing sites, identify potential resources, characterize the radiation environment and demonstrate new technology. The LRO was launched in 2009 and has completed the exploration phase of the mission, which lasted for 1-year after first turn-on has been completed and we are now in the science phase of the mission. The LOLA instrument is the first multi-beam altimeter system in space, a diffractive optical element (DOE) at the exit aperture of the transmit telescope produced five beams that illuminated the lunar surface. For each beam, LOLA measures time of flight.(range), pulse spreading (surface roughness), and transmit/return energy (surface reflectance). With its two-dimensional spot pattern, LOLA unambiguously determines slopes along and across the orbit track. Analysis of the data at cross-over points from multiple orbital tracks will also provide insight on the gravitational field of the moon and its center of mass. The LOLA laser transmitter, uses only the master oscillator from the GLAS modular design, is a diode pumped, Cr:Nd:YAG slab with passive q-switch and a cross-Porro resonator configuration. The laser transmitter consists of a beryllium (Be) flight laser bench housing two oscillators (a primary oscillator and a cold spare), an 18X transmit beam expander and a DOE [11].

The Cloud-Aerosol Lidar with Orthogonal Polarization (CALIOP) instrument, launched in 2006, is the one of three lidar instrument in operation at this time. The diode pumped Nd:YAG lasers produced two color output at $1064 \mathrm{~nm}$ and $532 \mathrm{~nm}$ at a pulse repetition rate of $20 \mathrm{~Hz}$. The lasers are actively q-switched to provide a pulse width of about $20 \mathrm{nsec}$. Each laser generates $220 \mathrm{~mJ}$ of 1064 energy, which is frequency-doubled to produce $110 \mathrm{~mJ}$ of energy at each of the two wavelengths.

\section{Future Lidar Missions}

All of the spaceborne lasers to-date has been low repetition rate (few tens of $\mathrm{Hz}$ ), high energy (few to hundreds of $\mathrm{mJ}$ ) and relatively long pulse width (few ns) with overall efficiencies in the few percent range $(<5 \%)$. We are currently working on ICESat-2, which is a follow on mission to ICESat, with a launch date of 2016. The ICESat-2 mission uses the micropulse lidar approach in which high repetition rate laser pulses on order of few to ten's of $\mathrm{kHz}$ are used with multiple beam configuration and lower energy requirement (tens to hundreds of $\mu \mathrm{J}$ ) per beam and short pulse width ( 1 ns) [12]. This instrument requirement drives the laser transmitter design to continuous wave (CW) laser diode pumped solid-state laser approach with either passive or active q-switches to generate the high repetition rate pulses. The demand for short pulsewidth and near diffraction limited beam profile also narrowed the laser architectures choice to the MOPA design.

ICESat-2 is a tier-1 mission as recommended in the National Research Council (NRC) Earth Science Decadal Report published in 2007 [13]. In addition to ICESat-2, there are two other lidar missions that are under development at GSFC. The Lidar Surface Topography (LIST) is a recommended tier-3 program in the NRC report. The objective of the mission is to globally map the topography of the Earth's solid surface with $5 \mathrm{~m}$ spatial resolution and $10 \mathrm{~cm}$ vertical precision, as well as the height of overlying covers of vegetation, water, snow, and ice. This foundation data is fundamental to understanding, modeling and predicting processes in a diverse range of Earth science disciplines such as natural hazards and landscape evolution; carbon storage in vegetation and its exchange with the atmosphere; habitat quality and its response to disturbance; ice sheet mass balance and its response to climate change; changing sea ice contributions to ocean-atmosphere energy exchange; and storage and transfer of on-land water resources.

The most difficult requirement is to reliably detect the ground height beneath dense, closed-canopy forest cover, for . which a lidar with high dynamic range is essential. In order to meet these requirements we have formulated a highly efficient lidar measurement approach utilizing 1,000 push-broom, micropulse laser beams coupled with a photonsensitive, linear-mode detector array. In our LIST measurement approach, the lidar operates in the near infrared (NIR) where, relative to the visible, solar background noise is low, atmospheric transmission and land surface reflectance are high, and NIR laser transmitters do not require frequency doubling with the associated loss of efficiency. In our previous work $[14,15]$, we established that the most efficient approach is use of a micropulse laser 
optically divided into multiple beams and operating at a $10 \mathrm{kHz}$ pulse rate with a narrow, $1 \mathrm{nsec}$ pulse width. Each beam produces a $5 \mathrm{~m}$ diameter footprint and a few tens of signal photons are detected per laser fire and the output from a photon-sensitive, analog detector is digitized. Accumulating the signals from 7 along-track laser fires over a distance of $5 \mathrm{~m}$ yields a range histogram comprised of several hundred photons necessary for detection of the ground beneath dense canopies as well as characterization of the canopy height and vertical structure. In this approach, a laser system capable of delivering $100 \mu \mathrm{J}$ per pulse per beam in the 1000 -beam swath is required. This represents an average optical power of $1 \mathrm{~kW}$ or a prime power of $>8 \mathrm{~kW}$ assuming a wall plug efficiency (WPE) of $12 \%$ for the laser system. This is still manageable but any efficiency that is less than this will become unrealistic for the instrument. This overall laser system efficiency of $>12 \%$, significantly exceeding current generation laser altimeters, is necessary to minimize required power, mass, size, complexity and cost. One of the greatest challenges for the mission is the development of a highly efficient laser system that can reliably provide the energy necessary to meet the LIST mission requirements [16].

The other lidar under development is a tier-2 missions recommended by the NRC. The Active Sensing of $\mathrm{CO}_{2}$ Emissions over Nights, Days, and Seasons (ASCENDS) mission will be the first laser spectroscopy from space with the objective to profile aerosol and cloud for climate and water cycle; ocean color for open ocean biogeochemistry. We have been working on an active remote sensing approach using an erbium fiber amplifier based transmitter for atmospheric $\mathrm{CO}_{2}$ measurements in an overtone band near $1.57 \mu \mathrm{m}$ and initial horizontal path measurements to $<1 \%$ precision. We are investigating different approaches to scale this laser transmitter for space deployment $[17,18]$.

\section{Conclusions}

Over the next two decades, the number of space based laser missions for mapping, spectroscopy, remote sensing and other scientific investigations will increase several folds. The demand for high wall-plug efficiency $(>15 \%)$ laser systems that can reliably operate over the life of a mission will be high. We are actively developing high efficiency laser systems that are suitable for spaceborne applications.

\section{References}

[1] Afzal, R.S., "Mars Observer Laser Altimeter: laser transmitter," Appl. Opt. 33, 3184-3188 (1994).

[2] Afzal, R.S.; Yu, A.W.; Dallas, J.L.; Melak, A.; Lukemire, A.T.L.; Ramos-Izqueirdo, L.; Mamakos, W., "The Geoscience Laser Altimeter System (GLAS) Laser Transmitter," Selected Topics in Quantum Electronics, IEEE Journal of, 13, 3, 511-536, (2007).

[3] Floyd E. Hovis, "Qualification of the Laser Transmitter for the CALIPSO Aerosol Lidar Mission", in Proc. of SPIE, 6100, 61001X (2006).

[4] Krebs, D.J., Anne-Marie Novo-Gradac, Steven X. Li, Steven J. Lindauer, Robert S. Afzal, and Anthony W. Yu, "Compact, passively Qswitched Nd:YAG laser for the MESSENGER mission to Mercury," Appl. Opt. 44, 1715-1718 (2005).

[5] Yu, A.W., A. M. Novo-Gradac, G. B. Shaw, S. X. Li, D. C. Krebs, L. A. Ramos-lzquierdo, G. L. Unger, and A. Lukemire, "Laser Transmitter for the Lunar Orbit Laser Altimeter (LOLA) Instrument," in Conference on Lasers and Electro-Optics/Quantum Electronics and Laser Science Conference and Photonic Applications Systems Technologies, OSA Technical Digest (CD) (Optical Society of America, 2008), paper CMQ2, (2008).

[6] Smith, D.E., et.al., "Mars Orbiter Laser Altimeter: Experiment summary after the first year of global mapping of Mars," J. Geophys. Res., 106, (E10), 23,689-23,722 (2001).

[7] Bufton, J.L., "Shuttle Laser Altimeter," Lasers and Electro-Optics, 1997. CLEO/Pacific Rim '97., Pacific Rim Conference on, 143-144, (1997).

[8] A compilation of the recent scientific results can be found in a special issue on 1CESat-1 in Geophysical Research Letters, 32, Numbers 21, 22 , and 23, (2005).

[9] Cavanaugh, J.F., et. al., "The Mercury Laser Altimeter Instrument for the MESSENGER Mission," Space Science Reviews, 131, $451-479$ (2007).

[10] Sjogrem, W.L., Apollo Laser Altimeter Analysis, Final Report, NASA JPL, (1975).

[11] Smith, J.G., L. Ramos-Izquierdo, A. Stockham, S. Scott, "Diffractive Optics for Moon Topography Mapping," Proc. SPIE, 6223, (2006).

[12] Yu, A.W.; et. al., "Space laser transmitter development for ICESat-2 mission," Paper 7578-9, Presented at SPIE Photonics West 2010, San Francisco, CA, (2010).

[13] Earth Science and Applications from Space: National Imperatives for the Next Decade and Beyond, National Research Council of the National academies, The National Academies Pres, Washington D.C., (2007).

[14] Callahan, L. et al., Advanced Mission Concept Study for LIST, Goddard Space Flight Center for NASA Headquarters, unpublished., (2010). [15] Harding, D. J. et al., "The Slope Imaging Multi-Polarization Photon-Counting Lidar: An advanced Technology Airborne Laser Altimeter," NASA Earth Science Technology Forum 2010, Paper A7P3., (2010).

[16] Yu, A.W., et. al., "Efficient Swath Mapping Laser Altimetry Demonstration," Paper G23A-0654, Presented at AGU Fall Meeting 2009, San Francisco, CA (2009).

[17] Abshire, J. B.; Harding, D. J.; Sun, X.; Krainak, M. A.; Shuman, C. A.; Dabney, P. W., "Space-based Swath Mapping Laser Altimeter for Cryospheric and Vegetation Mapping," American Geophysical Union, Fall Meeting, abstract \#G22A-05, (2007).

[18] Abshire, J.B., H. Riris, G.R. Allan, C.J. Weaver, J. Mao, X. Sun, W.E. Hasselbrack, S.R. Kawa and S. Biraud, "Pulsed airborne lidar measurements of atmospheric CO2 column absorption," Tellus-B, 62B, 770-783 (2010). 